\title{
A dose escalating phase I study of GLPG0187, a broad spectrum integrin receptor antagonist, in adult patients with progressive high-grade glioma and other advanced solid malignancies
}

\author{
Geert A. Cirkel ${ }^{1}$ - Bojana Milojkovic Kerklaan ${ }^{2} \cdot$ Frédéric Vanhoutte $^{3}$. \\ Annegret Van der $\mathrm{Aa}^{3}$ - Giocondo Lorenzon ${ }^{4}$. Florence Namour ${ }^{4}$. Philippe Pujuguet ${ }^{4}$. \\ Sophie Darquenne ${ }^{4} \cdot$ Filip Y. F. de Vos ${ }^{1}$ - Tom J. Snijders ${ }^{5}$ Emile E. Voest ${ }^{1,7}$ • \\ Jan H. M. Schellens ${ }^{2}$ • Martijn P. Lolkema ${ }^{1,6}$
}

Received: 4 December 2015 / Accepted: 28 December 2015 / Published online: 20 January 2016

(C) The Author(s) 2016. This article is published with open access at Springerlink.com

Summary Background Integrin signaling is an attractive target for anti-cancer treatment. GLPG0187 is a broad spectrum integrin receptor antagonist (IRA). GLPG0187 inhibited tumor growth and metastasis in mouse models. Methods We aimed to determine the Recommended Phase II Dose (RP2D) and to assess safety and tolerability of continuous i. $\mathrm{v}$. infusion in patients with advanced malignant solid tumors. Anticipated dose levels were 20, 40, 80, 160, 320, and $400 \mathrm{mg} /$ day in a modified $3+3$ design. Plasma concentrations of GLPG0187 were assessed to characterize the pharmacokinetics (PK). C-terminal telopeptide of type I collagen (CTX) was used as pharmacodynamics marker. Results Twenty

Electronic supplementary material The online version of this article (doi:10.1007/s10637-015-0320-9) contains supplementary material, which is available to authorized users.

Martijn P. Lolkema

m.lolkema@erasmusmc.nl

1 Department of Medical Oncology, University Medical Center Utrecht, Heidelberglaan 100, 3584 CX Utrecht, The Netherlands

2 Department of Clinical Pharmacology, Netherlands Cancer Institute, Plesmanlaan 121, 1066 CX Amsterdam, The Netherlands

3 Galapagos NV, Gen. De Wittelaan L11A3, 2800 Mechelen, Belgium

4 Galapagos SASU, 102 Avenue Gaston Roussel, 93230 Romainville, France

5 Brain Center Rudolf Magnus, Department of Neurology and Neurosurgery, University Medical Center Utrecht, Heidelberglaan 100, 3584 CX Utrecht, The Netherlands

6 Present address: Erasmus MC Kanker Instituut, Groene Hillededijk 301, Room G4-51, 3075 EA Rotterdam, The Netherlands

7 Present address: Netherlands Cancer Institute, Plesmanlaan 121, PO box: 90203, 1006 BE Amsterdam, The Netherlands patients received GLPG0187. No dose limiting toxicities (DLTs) were observed. The highest possible and tested dose was $400 \mathrm{mg} /$ day. Fatigue was the most frequently reported side effect (25\%). Recurrent Port-A-Cath-related infections and skin toxicity suggest cutaneous integrin inhibition. No dose-dependent toxicity could be established. PK analysis showed a short average distribution $(0.16 \mathrm{~h})$ and elimination (3.8 h) half-life. Continuous infusion resulted in dose proportional PK profiles. We observed decreases in serum CTX levels independent of the dose given, suggesting target engagement at the lowest dose level tested. Single agent treatment did not result in tumor responses. Conclusions GLPG0187 was well tolerated with a dose-proportional PK profile upon continuous infusion. No formal maximal tolerated dose could be established. GLPG0187 showed signs of target engagement with a favourable toxicity profile. However, continuous infusion of GLPG0187 failed to show signs of monotherapy efficacy.

Keywords Integrin · Antagonist · Glioma · Phase 1 . GLPG0187

\section{Introduction}

Integrin signaling plays an important role in cancer biology providing a strong rationale to pursue integrin receptor antagonists (IRA) as therapeutic agents in cancer patients [1, 2]. Integrin receptors are heterodimeric cell surface molecules that act as adhesion molecules connecting the cytoskeleton to the extracellular matrix. Moreover they are involved in activating intracellular signaling pathways, actin cytoskeleton remodelling, three-dimensional cell growth and metastatatic 
organotropism [3, 4]. Integrin-mediated signaling is implicated in modulation of well-known cancer-related pathways such as the TGF-beta pathway in glioblastoma and the Rho-Rac pathway $[2,5,6]$.

Cilengitide is the most advanced IRA in clinical development. Cilengitide showed signs of efficacy without significant additive toxicity both as single agent and combined with radiation and temozolomide in patients with glioblastoma multiforme (GBM) [7-9]. Unfortunately cilengitide failed to live up to its promise in a phase III clinical trial when combined with standard treatment in GBM and further clinical development seems unlikely [10-12]. When compared to cilengitide, GLPG0187 has a stronger nanomolar affinity for a broader panel of RGD (Arg-Gly-Asp) integrin receptors $(\alpha \mathrm{v} \beta 1, \alpha \mathrm{v} \beta 3, \alpha \mathrm{v} \beta 5, \alpha \mathrm{v} \beta 6, \alpha \mathrm{v} \beta 8$ and $\alpha 5 \beta 1$; supplementary table $\mathrm{S} 1$ and $[13,14])$. In preclinical models GLPG0187 significantly inhibited angiogenesis both in vivo and in vitro, osteoclastogenesis in vitro, and bone loss in vivo [15]. In mouse cancer models GLPG0187 inhibited de novo formation and progression of bone and visceral metastases in prostate cancer and breast cancer [13, 14, 16, 17]. We hypothesized that GLPG0187, a more potent and broader spectrum IRA when compared to cilengitide, may improve the anti-tumor efficacy of IRA therapy. Therefore a phase I dose escalation study was initiated to investigate the safety and tolerability of GLPG0187 when administered intravenously in end-stage cancer patients. In healthy volunteers, GLPG0187 was rapidly eliminated after oral administration with a terminal half-life of about 5-6 h [18]. To ensure continuous target inhibition despite its relatively short half-life GLPG0187 was administered as a continuous i.v. infusion in this study. We aimed to determine a safe dose in cancer patients and to determine the pharmacokinetics (PK), pharmacodynamics (PD) and evaluate preliminary signs of efficacy.

\section{Materials and methods}

\section{Patient selection}

Patients, aged over 18 years, with pathologically confirmed advanced or metastatic malignant solid tumors refractory to standard therapy or for whom no standard treatment options were available were eligible for participation. Additional inclusion criteria included: written informed consent, measurable disease according the Response Evaluation Criteria In Solid Tumors (RECIST) version 1.1 [19], Eastern Cooperative Oncology Group (ECOG) performance status of 0-2, estimated life expectancy of at least 12 weeks and no previously incurred anticancer therapy related toxicities higher than grade 2. Patients were considered ineligible if there was less than 4 weeks since their last anticancer therapy (less than 6 weeks for nitrosoureas and mitomycin C) or they were previously treated with IRAs. Additional exclusion criteria were: chronic treatment with corticosteroids equivalent to $10 \mathrm{mg}$ methylprednisolone per day or more, current or recent (within 30 days) treatment with another investigational drug or participation in another investigational study, clinically symptomatic or progressive brain or leptomeningeal metastases, major surgical procedure within 28 days before first dose, congestive heart failure (NYHA class 3 or 4), clinical significant cardiac arrhythmias, signs and symptoms of relevant cardiovascular disease, known hypersensitivity to any of the study drugs and significant medical conditions possibly interfering with patient compliance or safe study participation.

Female patients with reproductive potential were only eligible with a negative pregnancy test obtained less than 7 days before first dose and if an adequate contraceptive method was used while on study. There were no restrictions in concomitant medication.

The study was centrally approved by the ethics committee of the University Medical Center Utrecht and was conducted according to the Declaration of Helsinki and Good Clinical Practice guidelines. Written informed consent was obtained from all patients. The study was registered on clinicaltrials. gov (NCT01313598).

\section{Investigational agent}

GLPG0187 supply was controlled by Galapagos SASU (Romainville, France) and was delivered to participating sites as a $35 \mathrm{mg} / \mathrm{ml}$ injectable solution in type 1 clear glass vials. A $40 \%$ HP- $\beta-C D$ (Kleptose ${ }^{\circledR}$ ) injectable solution manufactured by Roquette pharma (Lestrem, France) was used to improve solubility of GLPG0187. Depending on dose level, various amounts of GLPG0187 were dissolved in HP- $\beta$-CD injectable solution and saline and administered by continuous infusion to patients at the recommended infusion rate. The amount of HP- $\beta$-CD needed to prepare a $400 \mathrm{mg} /$ day GLPG0187 solution (around $8 \mathrm{~g}$ /day) was considered the maximum acceptable dose in humans based on the clinical experience with itraconazole solved in HP- $\beta$-CD. No dose escalation beyond $400 \mathrm{mg}$ /day was planned within this study. After preparation, the solution was stored at room temperature protected from daylight for a maximum of 7 days.

\section{Study design}

This study was performed as a multicenter, open-label, dose-escalation, phase I study. Patients were accrued in the University Medical Center Utrecht and The Netherlands Cancer Institute. Decisions regarding dose escalation were made by using a modified $3+3$ dose escalation design. To reduce the number of patients treated at possible suboptimal dose concentrations, only 2 evaluable patients were assigned in the first two cohorts. 
A small wearable infusion pump (Pega plus infusion pump, Venner Medical, Ecublens,

Switzerland) enabled continuous GLPG0187 infusion at home through a Port-A-Cath (PAC) or peripherally inserted central catheter (PICC). Renewals of the infusion pumps and bags were performed at weekly hospital visits with at the higher dose levels additional renewals at home by specialized nurses. With these measures continuous infusion was possible with as little as possible impact on participants daily lives and wellbeing.

Patients in the first cohort received a starting dose of $20 \mathrm{mg} /$ day which was chosen based on results from a preceding healthy volunteer study [18]. The anticipated sequential dose escalations were 20,40, 80, 160, 320 and $400 \mathrm{mg}$ /day. Intrapatient dose-escalations were not allowed. Dose reductions or interruptions were allowed after cycle 1 which equals the dose limiting toxicity (DLT) window.

On day 1 of cycle 1, a single daily dose of GLPG0187 was administered at a constant infusion rate for a period of 1 hour after which the patient was followed for 24-h to assess the PK profile. On day 8 of cycle 1 continuous infusion was initiated at the assigned dose level for 21 days. Thereafter, treatment was continued uninterrupted in 21-day cycles until disease progression, occurrence of a DLT, unacceptable toxicity, death, poor study compliance or withdrawal of informed consent.

A DLT was defined as one of the following adverse events (AEs) considered related to GLPG0187 occurring within the first cycle of 28 days: grade 4 neutropenia lasting $\geq 7$ consecutive days, febrile neutropenia (defined as absolute neutrophil count (ANC) $\leq 1000$ cells per $\mu \mathrm{L}$ and fever $\geq 38.5^{\circ} \mathrm{C}$ ) or documented infection $\geq$ grade 3 with $\mathrm{ANC} \leq 1000$ cells $/ \mu \mathrm{L}$, grade 4 thrombocytopenia, thrombocytopenia requiring platelet transfusion, or bleeding requiring medical intervention, alanine aminotransferase (ALT) or aspartate aminotransferase (AST) $>5 \times$ upper limit of normal (ULN) $(>7.5 \times$ ULN in case of liver metastases) for greater than 14 days, ALT or AST $>5 \times \mathrm{ULN}(>7.5 \times \mathrm{ULN}$ in case of liver metastases) co-occurring with a total bilirubin of $>2.5 \times$ ULN (not explained by obstruction) regardless of duration, non-hematological toxicity $\geq$ grade 3. GLPG0187-related nausea, vomiting, and diarrhoea were only considered DLTs if they persisted at $\geq$ grade 3 for $>3$ days despite adequate supportive care measures.

The Maximum Tolerated Dose (MTD) for this study was defined as the dose level below the dose level at which $\geq 2$ patients in a dose cohort experienced a DLT within the DLT observation period. The resulting recommended phase II dose (RP2D) was defined as the MTD or the highest tested dose which is tolerable and safe.

\section{Safety and efficacy assessments}

After signing informed consent, patients were screened for eligibility. Screening assessments were performed within 14 days of the first dose.
Safety was assessed weekly by means of physical examination, weight, vital signs, ECOG performance status, laboratory evaluations (hematology, biochemistry and urinalysis), electrocardiograms, and recording of concurrent illness/ therapy and AEs throughout the study course. An AE was defined as appearance of any (or worsening of any preexisting) undesirable sign, symptom or medical condition occurring after signing the informed consent, whether related to treatment or not. Toxicity was graded according to the National Cancer Institute Common Terminology Criteria for Adverse Events (NCI-CTCAE) version 4.03. For each AE an absent, unlikely, possible, probable or certain relationship with GLPG0187 was assessed by the local investigator.

Preliminary efficacy was measured bi-cyclic and at end of treatment by CT scan, MRI, or a bone scan following RECIST 1.1 [19]. Recent literature has highlighted the need for better criteria for response assessment in high-grade gliomas, and the Response Assessment in Neuro-oncology (RANO) group has published new MRI-based response criteria [20]. For this reason, we evaluated all high-grade glioma patients according to RANO criteria. Concordance between RANO and RECIST evaluation of all gliomas was $100 \%$.

\section{Pharmacokinetic and pharmacodynamic methods}

PK blood samples were collected at baseline and on day 1 of cycle 1 at $0.5,1,1.5,2,4,6,8$ and $24 \mathrm{~h}$ after start of the single first dose. Additional samples were obtained on day 8, 15, 22 and 28 of cycle 1 . PK samples were analyzed for determination of GLPG0187 plasma levels by a validated liquid chromatography-mass spectrometry methods at AtlanBio (Saint-Nazaire, France). PK parameters in plasma such as maximum concentration $\left(\mathrm{C}_{\max }\right)$, Area Under the Curve (AUC), total plasma clearance, steady state volume of distribution $\left(\mathrm{V}_{\mathrm{ss}}\right)$ and distribution and elimination half-lives $\left(\mathrm{t}_{1 / 2 \mathrm{lbd} 1}\right.$ and $t_{1 / 2 \mathrm{lbdz}}$ ) were calculated, as well as dose standardized parameters $\left(\mathrm{C}_{\max } /\right.$ dose and $\mathrm{AUC} /$ dose $)$.

Integrin signaling is crucial for bone resorption by osteoclasts $[21,22]$. In a study performed by van der Horst et al. GLPG0187 inhibited osteoclastic bone resorption in mice significantly [15]. In addition, GLPG0187 was shown to significantly reduce CTX (C-terminal telopeptide of type I collagen) levels when compared with placebo in a phase I healthy volunteer study [18]. Therefore CTX, a well-established marker for bone turn-over, was adopted as surrogate PD marker in this study [23]. CTX serum levels were measured by ELISA, according to the manufacturer instructions (CrossLaps, Immuno Diagnostic Systems, ref. AC-02F1).

CTX levels were measured in blood samples collected at baseline and on day 1 of cycle 1 at 1, 2, 4, 6, 8 and $24 \mathrm{~h}$ after start of the first single dose. Additional samples were obtained on day $8,15,22$ and 28 of cycle 1 . These samples were obtained in the morning in a fasting state. 


\section{Statistical methods}

Study results were obtained by analyzing the safety population which contains all patients who received at least 1 dose of GLPG0187. Results were summarized descriptively and if applicable plotted by dose level over time. CTX levels at different time points were compared by using a Wilcoxon Signed Rank Test.

\section{Results}

\section{Patients}

Twenty patients received GLPG0187, between 22nd of March 2011 and 10th of April 2013. Patient characteristics are depicted in Table 1. Fifteen patients completed cycle 1 and were considered evaluable for DLT assessment. Patients with progressive high-grade glioma were most commonly included (40\%, GBMs) based on pre-clinical and early phase clinical data available [24, 7, 25].

\section{Dose escalation and safety}

No DLTs were observed in any cohort. The absence of DLTs resulted in an undisturbed dose escalation scheme towards the final planned cohort of $400 \mathrm{mg} / \mathrm{day}$. No MTD could be established.

GLPG0187 showed a tolerable toxicity profile in this study. The incidence of at least possibly related AEs per cohort is summarized in Table 2. Most frequently observed toxicities were fatigue ( 5 patients, $25 \%$ ) and skin related adverse events ( 5 patients, $25 \%$ ). Twenty-three AEs were considered possibly related and 6 probably related to GLPG0187. All but two AEs are reported only once. During the study, 14 (70\%) patients experienced a total of 23 serious adverse events (SAE). Only one SAE was considered possibly related (fatigue). All other SAEs were assessed as unlikely or not-related.

All toxicity seemed manageable and did not lead to dose reductions or dose interruptions. No clear relationship was observed between GLPG0187 dose level and the occurrence of AEs or laboratory abnormalities.

\section{Pharmacokinetic data}

After intravenous infusion, GLPG0187 was rapidly distributed and eliminated as illustrated in Fig. 1a. The PK profile was dose proportional over the 20 to $400 \mathrm{mg}$ /day dose range when infused continuously (Fig. 1b). PK parameters per cohort are displayed in Table 3. GLPG0187 showed a moderate total plasma clearance (average: $40.1 \mathrm{~L} / \mathrm{h}$ ) and short distribution and elimination half-lives of on average 0.16 and $3.8 \mathrm{~h}$,
Table 1 Patient demographics

\begin{tabular}{lc}
\hline & Total $(N=20)$ \\
& $\mathrm{N}(\%)$ \\
\hline Age (years) & \\
Mean (SD) & $56.4(11.9)$ \\
Median (range) & $58,5(35-76)$ \\
Gender & \\
Male & $14(70.0)$ \\
Female & $6(30.0)$ \\
ECOG & \\
0 & $4(20.0)$ \\
1 & $11(55.0)$ \\
2 & $5(25.0)$ \\
Ethnicity & $19(95.0)$ \\
Caucasian/white & $1(5.0)$ \\
Black & \\
Primary tumor & \\
High-grade gliomas & $1(5)$ \\
$\quad$ Glioblastoma multiforme & \\
Anaplastic oligodendroglioma & \\
High grade astrocytoma & \\
Colorectal carcinoma & $1(25)$ \\
Adenocarcinoma of Unknown Primary & $2(10)$ \\
Adenoid cystic carcinoma & $1(5)$ \\
Cholangiocarcinoma & $3(15)$ \\
Endometrial cancer & $1(5)$ \\
Nosopharynxcarcinoma & $1(5)$ \\
Ocular Melanoma & $1(5)$ \\
Osteosarcoma & $1(5)$ \\
Urothelial cell carcinoma & $1(5)$ \\
\hline
\end{tabular}

$N$ number of patients, ECOG Eastern Cooperative Oncology Group performance status

${ }^{\text {a }}$ Secondary form, from low grade astrocytoma

respectively. GLPG0187 plasma concentration was maintained during the PK sampling period of 21 days while receiving continuous i.v. infusion (Fig. 1b).

\section{Effects on bone resorption marker CTX}

The effect of GLPG0187 treatment on CTX levels was measured in serum during the first cycle and is depicted in Fig. 2a/b. High intra- and interpatient variability in the CTX concentration measurements was observed. The presence of bone metastases in 3 patients was not explanatory for the variability observed. A Wilcoxon Signed Rank Test was conducted to compare CTX levels of the total study population at baseline to $2 \mathrm{~h}$ post infusion on cycle 1 day 1 . Additionally, the effect of continuous infusion was analyzed by comparing mean CTX levels at day 
Table 2 All and $\geq$ Grade 3 at least possibly GLPG0187-related AEs per dose cohort

\begin{tabular}{|c|c|c|c|c|c|c|c|c|c|c|c|c|c|c|}
\hline \multirow[b]{2}{*}{ Adverse event description $^{\mathrm{a}}$} & \multicolumn{2}{|c|}{$\begin{array}{l}20 \mathrm{mg} / \text { day } \\
(N=2)\end{array}$} & \multicolumn{2}{|c|}{$\begin{array}{l}40 \mathrm{mg} / \text { day } \\
(N=5)\end{array}$} & \multicolumn{2}{|c|}{$\begin{array}{l}80 \mathrm{mg} / \mathrm{day} \\
(N=4)\end{array}$} & \multicolumn{2}{|c|}{$\begin{array}{l}160 \mathrm{mg} / \mathrm{day} \\
(N=3)\end{array}$} & \multicolumn{2}{|c|}{$\begin{array}{l}320 \mathrm{mg} / \text { day } \\
(N=3)\end{array}$} & \multicolumn{2}{|c|}{$\begin{array}{l}400 \mathrm{mg} / \text { day } \\
(N=3)\end{array}$} & \multicolumn{2}{|c|}{$\begin{array}{l}\text { Total } \\
(N=20)\end{array}$} \\
\hline & All & $\geq$ Gr 3 & All & $\geq$ Gr 3 & All & $\geq$ Gr 3 & All & $\geq$ Gr 3 & All & $\geq$ Gr 3 & All & $\geq$ Gr 3 & All & $\geq$ Gr 3 \\
\hline Dry mouth & 1 & 0 & 0 & 0 & 0 & 0 & 0 & 0 & 0 & 0 & 0 & 0 & 1 & 0 \\
\hline Epidermolysis & 0 & 0 & 0 & 0 & 1 & 0 & 0 & 0 & 0 & 0 & 0 & 0 & 1 & 0 \\
\hline Fungal skin infection & 0 & 0 & 0 & 0 & 0 & 0 & 0 & 0 & 1 & 0 & 0 & 0 & 1 & 0 \\
\hline Herpes zoster & 0 & 0 & 0 & 0 & 1 & 0 & 0 & 0 & 0 & 0 & 0 & 0 & 1 & 0 \\
\hline Mucosal inflammation & 1 & 0 & 0 & 0 & 0 & 0 & 0 & 0 & 0 & 0 & 0 & 0 & 1 & 0 \\
\hline Rash & 0 & 0 & 1 & 0 & 0 & 0 & 0 & 0 & 0 & 0 & 0 & 0 & 1 & 0 \\
\hline Rash Maculo-papular & 1 & 0 & 0 & 0 & 0 & 0 & 0 & 0 & 0 & 0 & 0 & 0 & 1 & 0 \\
\hline Skin hyperpigmentation & 1 & 0 & 0 & 0 & 0 & 0 & 0 & 0 & 0 & 0 & 0 & 0 & 1 & 0 \\
\hline Arthralgia & 1 & 0 & 0 & 0 & 0 & 0 & 0 & 0 & 0 & 0 & 0 & 0 & 1 & 0 \\
\hline Conjunctival Haemorrhage & 0 & 0 & 0 & 0 & 0 & 0 & 0 & 0 & 0 & 0 & 1 & 0 & 1 & 0 \\
\hline Diarrhoea & 1 & 0 & 0 & 0 & 0 & 0 & 0 & 0 & 0 & 0 & 0 & 0 & 1 & 0 \\
\hline Dysgeusia & 0 & 0 & 0 & 0 & 0 & 0 & 0 & 0 & 0 & 0 & 1 & 0 & 1 & 0 \\
\hline Fatigue & 0 & 0 & 0 & 0 & 2 & 0 & 1 & 0 & 1 & 1 & 1 & 0 & 5 & 1 \\
\hline Headache & 0 & 0 & 0 & 0 & 1 & 0 & 0 & 0 & 0 & 0 & 0 & 0 & 1 & 0 \\
\hline Oedema peripheral & 0 & 0 & 0 & 0 & 0 & 0 & 0 & 0 & 1 & 0 & 0 & 0 & 1 & 0 \\
\hline Peripheral sensory neuropathy & 1 & 0 & 0 & 0 & 0 & 0 & 0 & 0 & 0 & 0 & 0 & 0 & 1 & 0 \\
\hline Pleural effusion & 0 & 0 & 0 & 0 & 0 & 0 & 0 & 0 & 1 & 0 & 0 & 0 & 1 & 0 \\
\hline Thrombosis & 0 & 0 & 0 & 0 & 1 & 0 & 0 & 0 & 0 & 0 & 0 & 0 & 1 & 0 \\
\hline Vasculitis & 0 & 0 & 1 & 0 & 0 & 0 & 0 & 0 & 0 & 0 & 0 & 0 & 1 & 0 \\
\hline ALT increased & 0 & 0 & 0 & 0 & 1 & 1 & 0 & 0 & 0 & 0 & 0 & 0 & 1 & 1 \\
\hline Anemia & 0 & 0 & 1 & 0 & 0 & 0 & 0 & 0 & 0 & 0 & 0 & 0 & 1 & 0 \\
\hline Blood albumin decreased & 0 & 0 & 2 & 0 & 0 & 0 & 0 & 0 & 0 & 0 & 0 & 0 & 2 & 0 \\
\hline Blood bilirubin increased & 0 & 0 & 1 & 0 & 0 & 0 & 0 & 0 & 0 & 0 & 0 & 0 & 1 & 0 \\
\hline Blood creatinine increased & 0 & 0 & 1 & 0 & 0 & 0 & 0 & 0 & 0 & 0 & 0 & 0 & 1 & 0 \\
\hline Total & 7 & 0 & 7 & 0 & 7 & 1 & 1 & 0 & 4 & 1 & 3 & 0 & 29 & 2 \\
\hline
\end{tabular}

ALT Alanine aminotransferase, $N$ number of patients

${ }^{a}$ Adverse events were evaluated using the National Cancer Institute Common Toxicity Criteria for Adverse Events, version 4.03

15 to day 8. A significant change in CTX level was observed $2 \mathrm{~h}$ after the single dose infusion on day 1 . The mean CTX level of the total study population was higher at baseline: $0.58 \mathrm{ng} / \mathrm{ml}$ (SD 0.39) versus $0.42 \mathrm{ng} / \mathrm{ml}$ (SD $0.32), p<0.0001$. At day 15 CTX levels were lower compared to day $8(p=0.007)$. No relationship between GLPG0187 dose and change in CTX concentration was observed within cycle 1.

\section{Efficacy}

Stable disease was observed in $3(15 \%)$ out of 20 patients. These 3 patients were treated at $20 \mathrm{mg} /$ day (patient with non-small cell lung cancer, stable disease during 14 weeks), $80 \mathrm{mg} /$ day (patient with GBM, stable disease during 19 weeks) and $160 \mathrm{mg}$ /day (patient with GBM, stable disease during 8 weeks). No tumor responses were observed.

\section{Discussion}

We performed a phase I, open-label, dose escalation study using GLPG0187 in patients with solid tumors. GLPG0187 was well-tolerated and displayed a predictable and dose proportional PK profile. The toxicity profile of GLPG0187 when given as a continuous iv infusion is mild at the maximal administered dose and we did not identify a maximal tolerated dose.

Although no directly related severe toxicity was observed we did observe PAC related infections in three out of six patients in the first two cohorts. They received GLPG0187 through a PAC system. As the median reported infection rate of totally implantable intravenous catheter devices within an immunosuppressed population approximates 0.2 per 1000 catheter days [26] we consider these events retrospectively as possibly related to GLPG0187. For all three patients the infection was evident at the skin location where the needle 
Fig. 1 a The graph depicts the mean plasma concentration of GLPG0187 over the first $6 \mathrm{~h}$ after GLPG0187 treatment start. GLPG0187 concentration observed at 8 and $24 \mathrm{~h}$ postinfusion was below the limit of quantification. b The graph depicts the mean plasma concentration of GLPG0187 over 14 days during continuous GLPG0187 i.v. infusion. Plasma concentrations on day 8 were below the level of quantification
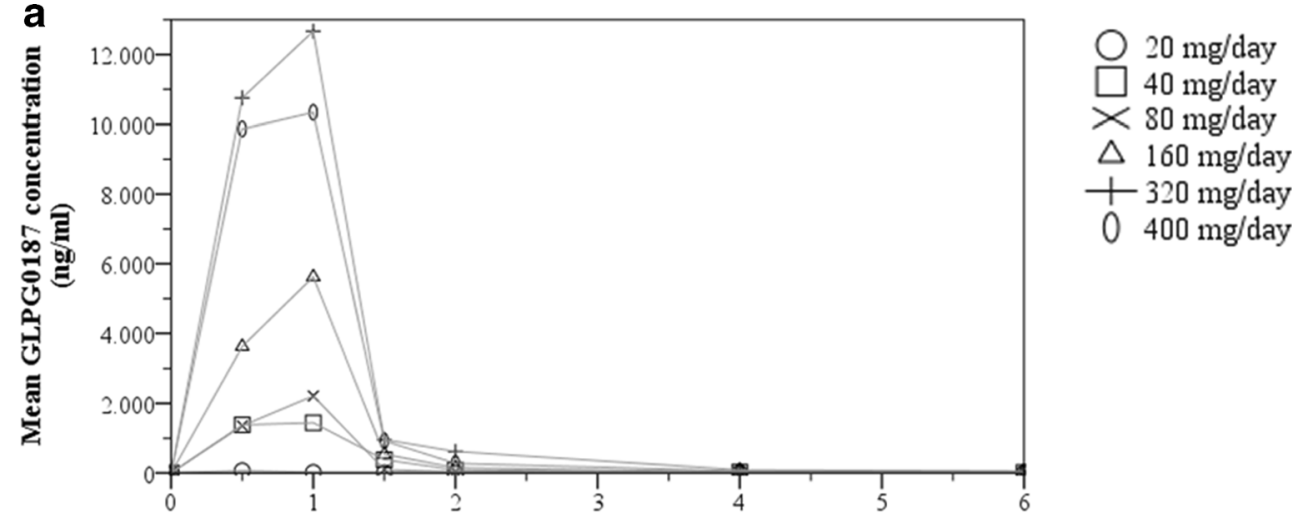

Time (hours)

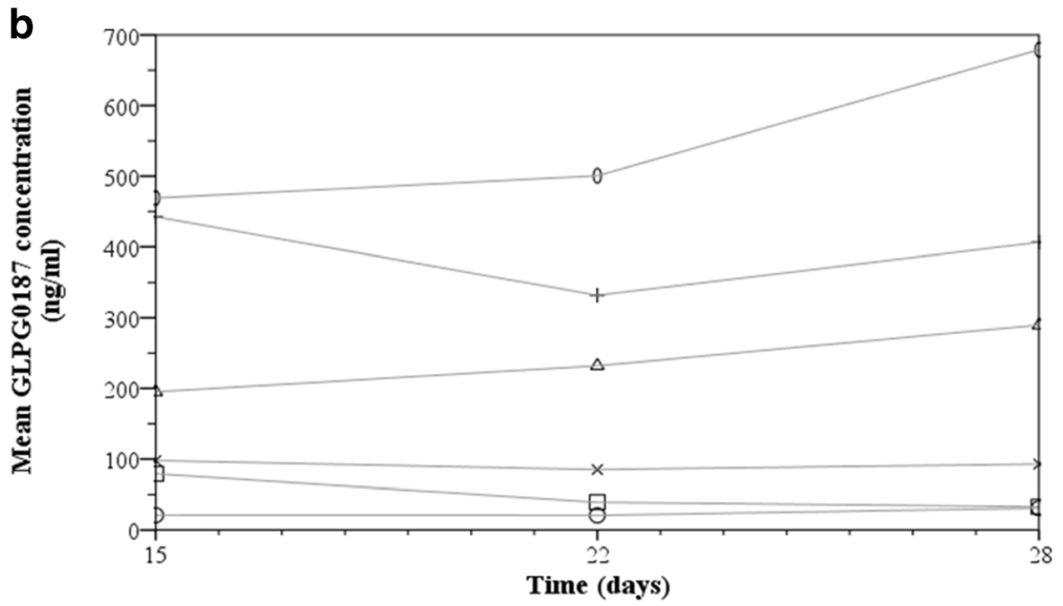

enters the skin. From cohort 3 onwards we switched to a PICC, where access to the system does not require repeated skin punctures. No catheter related infections were observed after the switch from PAC to PICC. These observations combined with the fact that skin toxicity was the most common side effect found suggest that skin integrity may be compromised in the presence of GLPG0187. The observed changes in
CTX levels indirectly suggest that GLPG0187 does inhibit integrin function. Despite the baseline variation we found a statistically significant decrease in CTX levels after GLPG0187 administration. Bone turnover, and associated markers including CTX, follow a circadian rhythm with peak levels at night and a decrease towards nadir in the afternoon $[27,28]$. All patients received their single dose on day 1 in the

Table 3 Mean PK (SD) parameters per dose cohort

\begin{tabular}{|c|c|c|c|c|c|c|}
\hline Parameter & $\begin{array}{l}20 \mathrm{mg} / \text { day } \\
(N=2)\end{array}$ & $\begin{array}{l}40 \mathrm{mg} / \text { day } \\
(N=5)\end{array}$ & $\begin{array}{l}80 \mathrm{mg} / \text { day } \\
(N=4)\end{array}$ & $\begin{array}{l}160 \mathrm{mg} / \text { day } \\
(N=3)\end{array}$ & $\begin{array}{l}320 \mathrm{mg} / \text { day } \\
(N=3)\end{array}$ & $\begin{array}{l}400 \mathrm{mg} / \text { day } \\
(N=3)\end{array}$ \\
\hline $\operatorname{AUC}_{(0 \text {-inf) }}(\mathrm{ng} \cdot \mathrm{h} / \mathrm{mL})$ & 422 & $1689(822)$ & $1866(387)$ & 4357 (1764) & $12,386(3951)$ & $10,114(6693)$ \\
\hline $\operatorname{AUC}_{(0-\text { inf)/dose }}(\mathrm{ng} \cdot \mathrm{h} / \mathrm{mL})$ & 21.1 & $47.3(18.4)$ & $23.3(4.8)$ & $27.2(11.0)$ & $38.7(12.3)$ & $25.3(16.7)$ \\
\hline $\mathrm{t}_{1 / 2, \mathrm{lbd} 1}(\mathrm{~h})$ & 0.182 & $0.180(0.097)$ & $0.117(0.045)$ & $0.165(0.060)$ & $0.148(0.022)$ & $0.140(0.030)$ \\
\hline $\mathrm{t}_{1 / 2, \mathrm{lbdz}}(\mathrm{h})$ & 2.41 & $2.98(1.01)$ & $3.92(1.27)$ & $4.68(0.35)$ & $5.07(0.26)$ & $3.44(1.11)$ \\
\hline $\mathrm{C}_{\max }(\mathrm{ng} / \mathrm{mL})$ & 391 & $1460(677)$ & $1751(396)$ & $3969(1511)$ & $11,490(3821)$ & $9592(6340)$ \\
\hline $\mathrm{C}_{\max / \text { dose }}(\mathrm{ng} / \mathrm{mL} \cdot \mathrm{mg})$ & 19.5 & $41.1(15.5)$ & $21.9(5.0)$ & $24.8(9.4)$ & $35.9(11.9)$ & $24.0(15.9)$ \\
\hline $\mathrm{CL}(\mathrm{L} / \mathrm{h})$ & 48.7 & $24.3(10.3)$ & $44.3(9.2)$ & $42.5(21.6)$ & $27.6(8.2)$ & $53.2(33.3)$ \\
\hline Vss (L) & 25.6 & $15.4(13.0)$ & $25.7(17.3)$ & $26.1(2.2)$ & $20.6(7.1)$ & $23.0(14.5)$ \\
\hline
\end{tabular}

$N$ number of patients, $A U C$ area under the curve, $\mathrm{t}_{1 / 2, \text { lbd } 1}$ distribution half- life, $\mathrm{t}_{1 / 2, \text { lbdz }}$ terminal elimination half- life, $\mathrm{C}_{\text {max }}$ maximum concentration, $C L$ clearance, Vss steady state volume of distribution 
Fig. 2 a Mean concentration of bone resorption marker CTX over the first $24 \mathrm{~h}$ after a single i.v. dose of GLPG0187. b Mean CTX concentration measured on day 8 , 15, 22 and 28. Continuous infusion of GLPG0187 was initiated on day 8

\section{a}

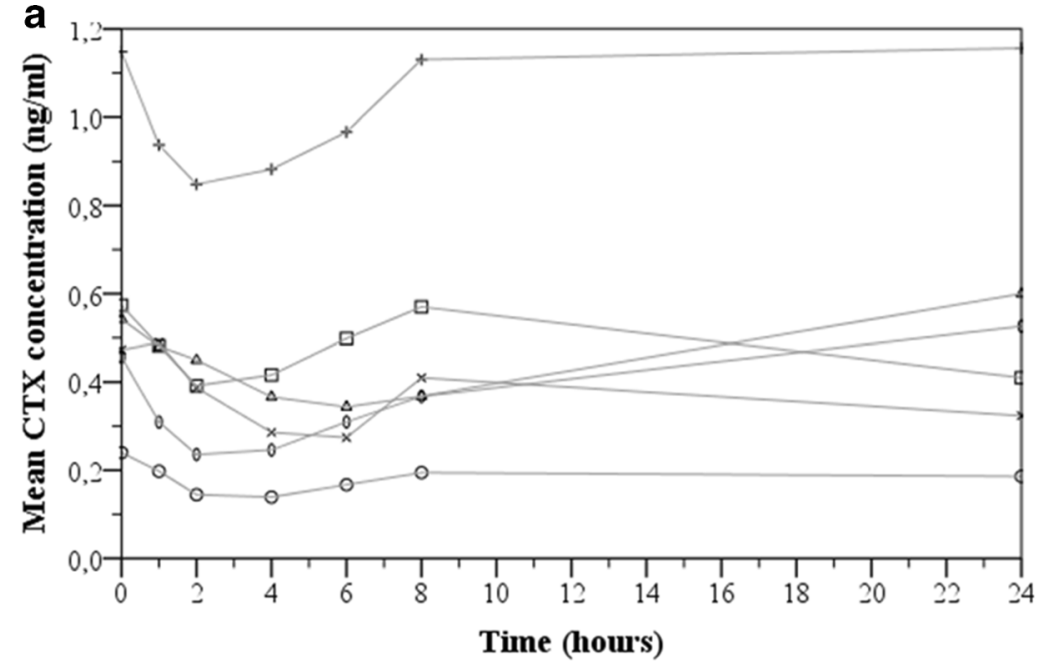

$20 \mathrm{mg} /$ day

$\square 40 \mathrm{mg} /$ day

$\times 80 \mathrm{mg} /$ day

$\triangle 160 \mathrm{mg} /$ day

$+320 \mathrm{mg} /$ day

$0400 \mathrm{mg} /$ day

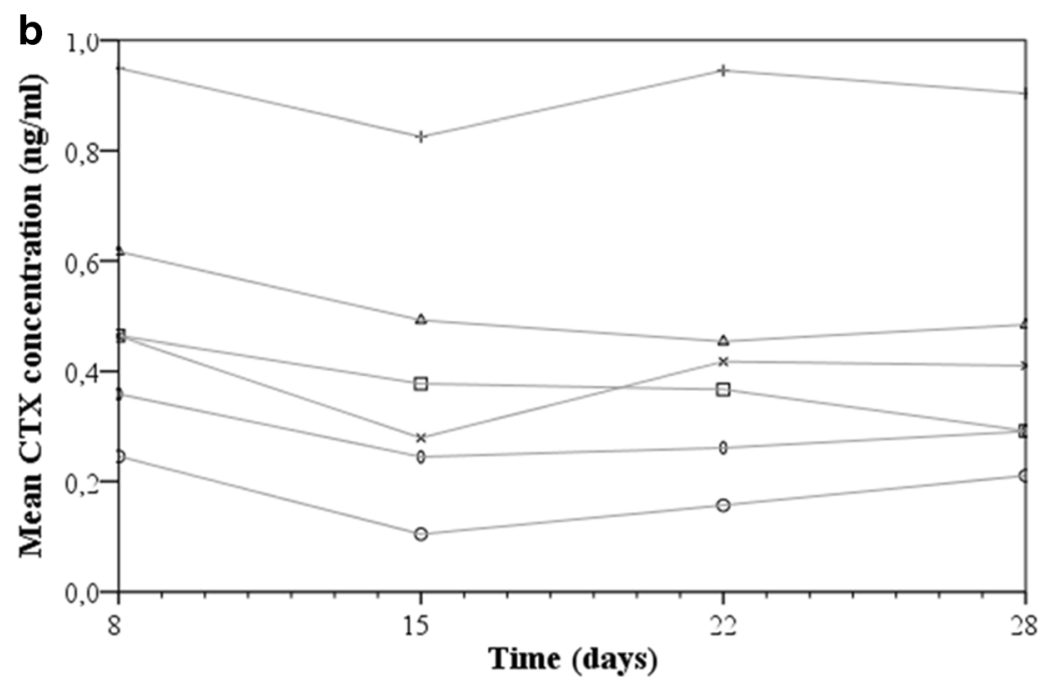

morning. Therefore, on day 1 we cannot exclude the possibility that CTX levels in our patients partially, if not completely, decreased by a physiological phenomenon rather than a PD effect. However, the clear decrease at day 15 cannot be explained in this manner and most likely represents a treatment effect. Thus, both toxicity and PD correlative markers suggest a treatment dependent inhibition of physiological integrin signaling.

During this study the development of cilengitide, the first generation IRA stopped due to a negative trial in patients with high-grade glioma $[10,11]$. These disappointing results for cilengitide may reflect either inherent inefficacy of cilengitide or problems with dose, scheduling and patient selection. Regarding dose and scheduling it appears that more could be less and intermittent scheduling could have more efficacy than more intensive schedules [12]. Here we explored the extreme of exposure driven therapy: continuous iv infusion. Although continuous exposure seems to be important in treatment targeting driving oncogenic pathways such as BRAF or EGFR it is questionable whether integrin signaling could be classified as such. Authors have proposed that inadequate exposure may in part explain the lack of efficacy of cilengitide [29]. Our data suggest that even continuous iv infusion of a more potent IRA does not result in the expected efficacy signals.

The development of IRAs in oncology has been largely disappointing and our study suggests that the lack of efficacy cannot easily be attributed to exposure, completeness of integrin signaling blockade or affinity issues of the first generation compounds. Indeed the pre-clinical models have failed to lead the way for rational drug design and rational trial design for IRAs. The data known for cilengitide in the clinical setting warrants a discontinuation of IRA development until novel more relevant combination strategies have been designed that can be tested in small proof-of-principle clinical trials.

Acknowledgments Cilengitide, used for a head-to-head comparison with GLPG0187 of relative binding affinities to RGD integrin receptors, was kindly provided to us by prof D. Cheresh (Moores, UCSD Cancer Center, LaJolla CA 92093). 
We thank Specialized Medical Services-oncology BV (SMSoncology) for managing the study as a clinical CRO.

\section{Compliance with ethical standards}

Funding Galapagos SASU.

Conflict of interest Florence Namour, Frédéric Vanhoutte, Annegret Van der Aa, Giocondo Lorenzon, Philippe Pujuguet and Sophie Darquenne are employees of Galapagos NV or Galapagos SASU.

Florence Namour, Frédéric Vanhoutte, Annegret Van der Aa and Giocondo Lorenzon also hold Galapagos NV stock options.

All other authors have no conflicts of interest to declare.

Ethical approval All procedures performed in studies involving human participants were in accordance with the ethical standards of the institutional and/or national research committee and with the 1964 Helsinki declaration and its later amendments or comparable ethical standards.

Open Access This article is distributed under the terms of the Creative Commons Attribution 4.0 International License (http://creativecommons. org/licenses/by/4.0/), which permits unrestricted use, distribution, and reproduction in any medium, provided you give appropriate credit to the original author(s) and the source, provide a link to the Creative Commons license, and indicate if changes were made.

\section{References}

1. Hoshino A, Costa-Silva B, Shen TL, Rodrigues G, Hashimoto A, Tesic Mark M, Molina H, Kohsaka S, Di Giannatale A, Ceder S, et al. (2015) Tumour exosome integrins determine organotropic metastasis. Nature 527:329-335

2. Desgrosellier JS, Cheresh DA (2010) Integrins in cancer: biological implications and therapeutic opportunities. Nat Rev Cancer 10:922

3. Zaman MH, Trapani LM, Sieminski AL, Mackellar D, Gong H, Kamm RD, Wells A, Lauffenburger DA, Matsudaira P (2006) Migration of tumor cells in 3D matrices is governed by matrix stiffness along with cell-matrix adhesion and proteolysis. Proc Natl Acad Sci U S A 103:10889-10894

4. Huttenlocher A, Horwitz AR (2011) Integrins in cell migration. Cold Spring Harb Perspect Biol 3:a005074

5. Roth P, Silginer M, Goodman SL, Hasenbach K, Thies S, Maurer G, Schraml P, Tabatabai G, Moch H, Tritschler I, et al. (2013) Integrin control of the transforming growth factor-beta pathway in glioblastoma. Brain 136:564-576

6. Silginer M, Weller M, Ziegler U, Roth P (2014) Integrin inhibition promotes atypical anoikis in glioma cells. Cell Death Dis 5:e1012

7. Nabors LB, Mikkelsen T, Hegi ME, Ye X, Batchelor T, Lesser G, Peereboom D, Rosenfeld MR, Olsen J, Brem S, et al. (2012) A safety run-in and randomized phase 2 study of cilengitide combined with chemoradiation for newly diagnosed glioblastoma (NABTT 0306). Cancer 118:5601-5607

8. Reardon DA, Fink KL, Mikkelsen T, Cloughesy TF, O'Neill A, Plotkin S, Glantz M, Ravin P, Raizer JJ, Rich KM, et al. (2008) Randomized phase II study of cilengitide, an integrin-targeting arginine-glycine-aspartic acid peptide, in recurrent glioblastoma multiforme. J Clin Oncol 26:5610-5617

9. Stupp R, Hegi ME, Neyns B, Goldbrunner R, Schlegel U, Clement PMJ, Grabenbauer GG, Ochsenbein AF, Simon M, Dietrich PY, et al. (2010) Phase I/IIa study of cilengitide and temozolomide with concomitant radiotherapy followed by cilengitide and temozolomide maintenance therapy in patients with newly diagnosed glioblastoma. J Clin Oncol 28:2712-2718

10. Stupp R, Hegi ME, Gorlia T, Erridge SC, Perry J, Hong YK, Aldape KD, Lhermitte B, Pietsch T, Grujicic D, et al. (2014) Cilengitide combined with standard treatment for patients with newly diagnosed glioblastoma with methylated MGMT promoter (CENTRIC EORTC 26071-22072 study): a multicentre, randomised, open-label, phase 3 trial. Lancet Oncol 15:1100-1108

11. Mason WP (2015) End of the road: confounding results of the CORE trial terminate the arduous journey of cilengitide for glioblastoma. Neuro Oncol 17:634-635

12. Nabors LB, Fink KL, Mikkelsen T, Grujicic D, Tarnawski R, Nam dH, Mazurkiewicz M, Salacz M, Ashby L, Zagonel V, et al. (2015) Two cilengitide regimens in combination with standard treatment for patients with newly diagnosed glioblastoma and unmethylated MGMT gene promoter: results of the open-label, controlled, randomized phase II CORE study. Neuro Oncol 17:708-717

13. Pujuguet $\mathrm{P}$, Berrocal E, Heckmann B, Wigerinck P, Lorenzon G, Clement-Lacroix P, Bormans G, Deroose C (2011) PP 87 molecular imaging demonstrates GLPG0187, a small-molecule integrin antagonist, binds to RGD-integrin receptors in vivo and is efficacious in tumor and metastasis models. Eur J Cancer 47:S28

14. Avraamides CJ, Garmy-Susini B, Varner JA (2008) Integrins in angiogenesis and lymphangiogenesis. Nat Rev Cancer 8:604-617

15. van der Horst G, van den Hoogen C, Buijs JT, Cheung H, Bloys H, Pelger RC, Lorenzon G, Heckmann B, Feyen J, Pujuguet P, et al. (2011) Targeting of alpha(v)-integrins in stem/progenitor cells and supportive microenvironment impairs bone metastasis in human prostate cancer. Neoplasia 13:516-525

16. Clement-Lacroix P, Berrocal E, Heckmann B, Wigerinck P, Lorenzon G, P P (2011) GLPG0187 inhibits progression of established bone metastasis and achieves maximum efficacy when combined with standard-of-care metastatic breast cancer treatments. Bone 48:S45

17. Zhao Y, Bachelier R, Treilleux I, Pujuguet P, Peyruchaud O, Baron R, Clement-Lacroix P, Clezardin P (2007) Tumor alphavbeta3 integrin is a therapeutic target for breast cancer bone metastases. Cancer Res 67:5821-5830

18. Lorenzon G, Gheyle L, Vets E, Namour F, Pujuguet P, ClementLacroix P, Wigerinck P, Vanhoutte F (2010) Abstract 1568: GLPG0187, a small molecule integrin antagonist, shows good safety and decrease in CTX levels in single ascending dose study. Cancer Res 70:1568

19. Eisenhauer EA, Therasse P, Bogaerts J, Schwartz LH, Sargent D, Ford R, Dancey J, Arbuck S, Gwyther S, Mooney M, et al. (2009) New response evaluation criteria in solid tumours: revised RECIST guideline (version 1.1). Eur J Cancer 45:228-247

20. Wen PY, Macdonald DR, Reardon DA, Cloughesy TF, Sorensen AG, Galanis E, DeGroot J, Wick W, Gilbert MR, Lassman AB, et al. (2010) Updated response assessment criteria for high-grade gliomas: response assessment in neuro-oncology working group. $\mathrm{J}$ Clin Oncol 28:1963-1972

21. Engleman VW, Nickols GA, Ross FP, Horton MA, Griggs DW, Settle SL, Ruminski PG, Teitelbaum SL (1997) A peptidomimetic antagonist of the alpha(v)beta3 integrin inhibits bone resorption in vitro and prevents osteoporosis in vivo. J Clin Invest 99:2284 2292

22. Davies J, Warwick J, Totty N, Philp R, Helfrich M, Horton M (1989) The osteoclast functional antigen, implicated in the regulation of bone resorption, is biochemically related to the vitronectin receptor. J Cell Biol 109:1817-1826

23. Rosen HN, Moses AC, Garber J, Iloputaife ID, Ross DS, Lee SL, Greenspan SL (2000) Serum CTX: a new marker of bone resorption that shows treatment effect more often than other markers because of low coefficient of variability and large changes with bisphosphonate therapy. Calcif Tissue Int 66:100-103 
24. Uhm JH, Dooley NP, Kyritsis AP, Rao JS, Gladson CL (1999) Vitronectin, a glioma-derived extracellular matrix protein, protects tumor cells from apoptotic death. Clin Cancer Res 5:1587-1594

25. Bello L, Francolini M, Marthyn P, Zhang J, Carroll RS, Nikas DC, Strasser JF, Villani R, Cheresh DA, Black PM (2001) Alpha(v) beta3 and alpha(v)beta5 integrin expression in glioma periphery. Neurosurgery 49:380-389

26. Bouza E, Burillo A, Muños P (2002) Catheter-related infections: diagnosis and intravascular treatment. Clin Microbiol Infect 8:265-274
27. Aoshima H, Kushida K, Takahashi M, Ohishi T, Hoshino H, Suzuki M, Inoue T (1998) Circadian variation of urinary type I collagen crosslinked C-telopeptide and free and peptide-bound forms of pyridinium crosslinks. Bone 22:73-78

28. Wichers M, Schmidt E, Bidlingmaier F, D K (1999) Diurnal rhythm of CrossLaps in human serum. Clin Chem 45:18581860

29. Tucci M, Stucci S, Silvestris F (2014) Does cilengitide deserve another chance? Lancet Oncol 15:e584-e585 